This is the peer reviewed version of the following article:

Liu J., Paradinas M., Heinke L., Buck M., Ocal C., Mugnaini V., Wöll C.. Film Quality and Electronic Properties of a

Surface-Anchored Metal-Organic Framework Revealed by using a Multi-technique Approach. ChemElectroChem, (2016). 3. : 713 - . 10.1002/celc. 201500486 ,

which has been published in final form at https://dx.doi.org/10.1002/celc. 201500486 . This article may be used for non-commercial purposes in accordance with Wiley Terms and Conditions for Use of Self-Archived Versions. 


\title{
Film quality and electronic properties of a surface-anchored metal-organic framework as revealed by a multi-technique
} approach

\author{
Jianxi Liu, ${ }^{[a]}$ Markos Paradinas, ${ }^{[b],[c]}$ Lars Heinke, ${ }^{[a]}$ Manfred Buck, ${ }^{[d]}$ Carmen Ocal, ${ }^{[b]}$ Veronica \\ Mugnaini, ${ }^{*[a],[e]}$ Christof Wöll ${ }^{\star[a]}$
}

\begin{abstract}
The virtually unlimited versatility and unparalleled level of control in the design of metal-organic frameworks (MOFs) has recently been shown to also entail a potential for applications based on the electrical and electronic properties of this rich class of materials. Since at present methods to provide reliable and reproducible contacts to MOF-materials are scarce, we have carried out a detailed, multi-technique investigation of an empty and loaded prototype MOF, HKUST-1. Epitaxial thin films of this material grown on a substrate using liquid-phase epitaxy have been studied by cyclic voltammetry (CV), atomic force microscopy (AFM), and quartz crystal microbalance (QCM), and their quality assessed. By using an ionic liquid (IL) as electrolyte it is shown that redox active molecules like ferrocene can be embedded in the pores, enabling to change the overall conductivity of the framework and to study the redoxchemistry of guest molecules inside the MOF.
\end{abstract}

Porous metal-organic frameworks (MOFs), also referred to as porous coordination polymers (PCPs), are crystalline hybrid materials formed by metal nodes and organic linkers held together by coordination bonds. ${ }^{[1]}$ The possibility to tailor their functionality either by the rational design of the organic ligands ${ }^{[2]}$ or by filling the framework's pores with functional nano-objects ${ }^{[3]}$ provides the basis for the continuously widening field of applications of MOFs, ${ }^{[4]}$ with electronics ${ }^{[5]}$ and batteries ${ }^{[6]}$ as rather recent extensions. Using electroactive molecules ${ }^{[7]}$ as linkers between the metal nodes or by doping the nanopores with electroactive guests, ${ }^{[3 a, 8]}$ MOFs are indeed extremely appealing materials for electronics and implementation in so-

[a] Dr. J. Liu, Dr. L. Heinke, Dr. V. Mugnaini, Prof. Dr. C. Wöll Institute of Functional Interfaces (IFG) Karlsruhe Institute of Technology (KIT)

76344 Eggenstein-Leopoldshafen (Germany)

E-mail: veronica.mugnaini@inl.int, christof.woell@kit.edu

[b] Dr. M. Paradinas, Prof. Dr. C. Ocal

Institut de Ciència de Materials de Barcelona (ICMAB-CSIC) Campus de la UAB

E-08193, Bellaterra (Spain)

[c] Present address: Catalan Institute of Nanoscience and Nanotechnology (ICN2) and the Barcelona Institute of Science and Technology, Campus de la UAB, Bellaterra (Spain)

[d] Prof. Dr. M. Buck

EaStCHEM School of Chemistry

University of St Andrews

North Haugh, St. Andrews KY16 9ST (United Kingdom)

[e] Present address: International Iberian Nanotechnology Laboratory (INL), 4715-330, Braga (Portugal)

Supporting information for this article is available on the WWW under http:// XXXXXXXXXXX. called MOFtronic devices. ${ }^{[9]}$ Although a huge potential is seen for MOFs in the field of electronics and also photovoltaics ${ }^{[10]}$ the prediction and understanding of the electronic and electrical properties are still in their incipiency due to the very limited number of studies reported so far. ${ }^{[11]}$ A major reason, which is also a bottleneck for the actual integration of MOFs into a device, is represented by the fabrication of supported MOF thin films exhibiting the reproducible quality as regards their intrinsic properties like conductivity and the diffusion constant governing the loading with guest molecules. ${ }^{[12]}$

Several strategies have been developed for fabricating MOFs as thin films. ${ }^{[13]}$ Among these, the liquid phase epitaxy $(\mathrm{LPE})^{[13 a, \mathrm{e}-\mathrm{h}]}$ appears as one of the most appropriate preparation methods to meet the requirements for electronic applications of MOFs. Through a stepwise growth of the MOF on a selfassembled monolayer (SAM) whose chemical functionality and/or packing density can be tailored at will, ${ }^{[14]}$ continuous crystalline layers on a surface are obtained, referred to as SURMOFs (surface-anchored metal-organic framework). The functionalization of the supporting substrate, the choice of the growth method and the number of growth cycles allows for a precise control over the film thickness and the crystallographic orientation of the MOF thin film. ${ }^{[13 a]}$ In addition, the LPE process allows the fabrication of hetero-multilayers in a straightforward fashion. ${ }^{[15]}$

For the device applications of MOFs, the following topics are of paramount interest: firstly, the preparation of robustly surface-anchored and defect-free thin films covering extended areas on the order of $\mathrm{cm}^{2}$; secondly, the identification of the most suitable techniques to characterize and monitor them. Indeed, the most widely used SURMOF characterization techniques (X-ray diffraction, XRD, and infrared reflection absorption spectroscopy, IRRAS) allow only the determination of the long-range crystalline order of MOF thin films and their chemical composition, respectively. However these methods are not very sensitive to the presence of pinholes and other structural defects that could unpredictably alter the electrical properties of the SURMOF film when attaching a top electrode. Imaging techniques such as atomic force microscopy (AFM) allow the observation of the surface, the determination of the film roughness and, eventually, the presence of defects, but only within a small $\left(\mu \mathrm{m}^{2}\right)$ area. ${ }^{[16]}$ To gain insight into defect densities on a $\mathrm{mm}^{2}$ or even $\mathrm{cm}^{2}$ area AFM measurements alone become extremely time-consuming.

Herein we will present cyclic voltammetry (CV), in combination with AFM, as a fast method to determine the quality of SURMOF films used as working electrodes in a home-made electrochemical cell with platinum wires as counter and 
reference electrodes (see Figure S1 in Supporting Information $(\mathrm{SI})$ ). Eventually, we will also show the suitability of cyclic voltammetry as characterization technique for SURMOF films loaded with a small electroactive molecule, ferrocene, and demonstrate how important information on the conduction mechanism can be deduced from the shape of the cyclic voltammogram.

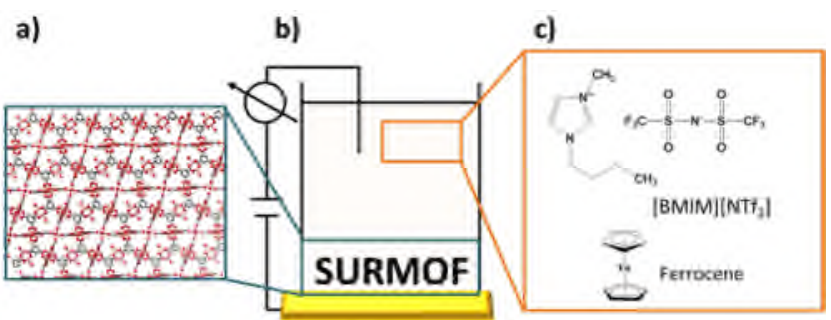

Figure 1. Schematic representation of the use of SURMOF as working electrodes in an electrochemical cell. a) Side view of HKUST-1 crystal structure grown along the [111] direction on a CMMT SAM. In red, oxygen; in blue, copper; in black, carbon. b) Schematic representation of the electrochemical cell. c) Molecular formula of the used ionic liquid (IL) and of ferrocene.

We have investigated the surface-anchored porous and crystalline HKUST-1 (also termed $\mathrm{Cu}_{3}(\mathrm{BTC})_{2}$, where BTC is 1,3,5-benzenetricarboxylic acid) ${ }^{[17]}$ grown by LPE on a gold electrode modified by SAMs.

In going beyond previous work reported by some of us ${ }^{[8 \mathrm{a}]}$ we have studied different types of SAMs to initiate the MOFgrowth. More specifically, CMMT (9-carboxy-10(mercaptomethyl)triptycine, SAM1), and TPMTA (4'carboxyterphenyl-4-methanethiol, SAM2) were chosen to functionalize the supporting Au-substrate. In both cases crystalline and oriented films (herein named SAM1/MOF, and SAM2/MOF, respectively) of different crystallographic orientation were obtained. ${ }^{[14]}$ Figure 1a shows the case of SAM1/MOF HKUST-1 crystalline films with the [111] direction parallel to the surface normal.

The electrochemical measurements were carried out in an aprotic ionic liquid (IL), 1-butyl-3-methylimidazolinium bis(trifluoronethylsulfonyl)-imide, [BMIM] $\left[\mathrm{NTf}_{2}\right]$ (Figure 1c), acting as solvent and supporting electrolyte. The ionic components of this IL do not interfere with the coordination bonds in the HKUST-1 SURMOF and preserve it from losing its crystalline structure (see Figure S2 in SI). Moreover, like several other known ionic liquids, ${ }^{[18]}$ the used $[\mathrm{BMIM}]\left[\mathrm{NTf}_{2}\right]$ is a good supporting electrolyte for $a$ ) its electrochemical inertness (i.e. its wide potential window which is larger than the one of the electroactive species under study) and $b$ ) its high conductivity.

Of course, the supporting electrolyte in the specific case of a cyclic voltammetry experiment has to be able to penetrate and diffuse in the pores of the SURMOF HKUST-1. Due to the rather small size of the [BMIM] $\left[\mathrm{NTf}_{2}\right]$, it is reasonable to assume that it will diffuse inside the pores of the SURMOF. Nevertheless, since this is a crucial point, we used a quartz crystal microbalance $(\mathrm{QCM})^{[12,19]}$ to verify this assumption.
In a QCM experiment, the changes in resonance frequency of an oscillating sensor crystal are monitored in real time. Thanks to the relationship between resonance frequency shift and adsorbed/trapped mass, information on the amount of material deposited on sensor can be calculated.

A straightforward experiment to investigate the uptake of a guest molecule by a SURMOF-coated QCM sensor consists of letting a solvent (such as ethanol, EtOH) flow over the QCM sensor and then exchanging it with the solution of the guest molecule whose uptake by the SURMOFs is to be studied (ferrocene in IL, in the specific case). In the case of IL, however, this is not feasible in the way described above: changes in frequency measured by QCM are very sensitive to variations in the viscosity ${ }^{[20]}$ and the IL used has a much higher viscosity and density ${ }^{[18,21]}$ than the ethanol. Indeed, when the sample was exposed to the IL solution, the mass changes recorded by the QCM could not be unambiguously ascribed to the IL uptake.

Therefore, in order to investigate whether there is an IL uptake by the HKUST-1 SURMOF, we proceeded as follows: first, the sample was activated under argon gas flow at $60^{\circ}$ for at least 6 hours. Then, pure liquid ethanol was flowed through the activated SURMOF HKUST-1 (Figure 2, red part). After 150 minutes, the surface was exposed to the IL/EtOH (96:4, volume ratio) solution. When the solvent was exchanged from $\mathrm{EtOH}$ to IL/EtOH (Figure 2, green part), an increase of the SURMOF mass of $22 \mu \mathrm{g} / \mathrm{cm}^{2}$ was calculated using the Sauerbrey equation, ${ }^{[22]}$ which is a hint that IL diffuses into the SURMOF. It is important to stress, that the Sauerbrey equation is only correct under the assumption of a thin film approximated as rigid and for which the energy dissipation can be neglected. In the experiments here presented, however, by moving from gas to liquid phase (and vice versa) we significantly change the viscosity of the environment in contact with the sensing crystal, and hence we record a rather high dissipation change (data not shown). This means the determined values of mass changes (Fig. 2), calculated from the frequency changes, serve only as

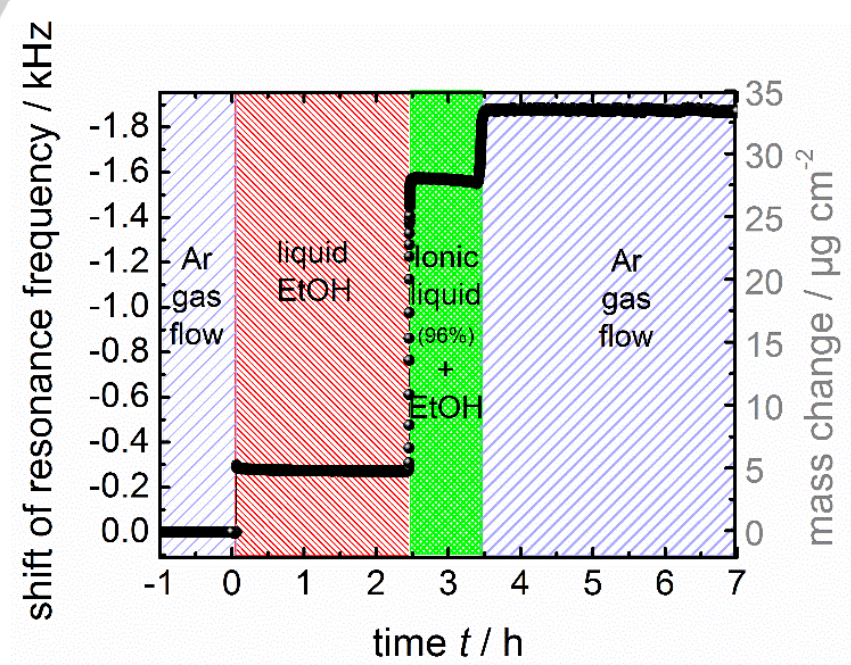

rough estimation of mass uptake.

Figure 2. Ionic liquid uptake by HKUST-1 SURMOF measured by QCM. The shift in resonance frequency is plotted on the left $y$-axis, while the corresponding calculated mass change, estimated by the Sauerbrey equation, is plotted on the right $y$-axis. 
When the argon is switched on, we experimentally observe a small change in frequency that corresponds to a calculated mass increase of about $4 \mu \mathrm{g} \mathrm{cm}^{-2}$. A possible reason for the change in the resonance frequency and hence the associated mass increase is the following, according to the authors' opinion: as soon as the argon is switched on, $\mathrm{EtOH}$ molecules forming the IL:EtOH mixture evaporate, while the IL does not due to its low vapor pressure. This results in an increased IL-ratio and an increased density of the mixture inside the QCM-cell (and then in the SURMOF). The density and viscosity of IL, higher than the ones of its ethanolic mixture, is believed to be responsible for the mass increase.

A further experiment to prove that the IL can be loaded into the pores of the SURMOFs is shown in Figure S3. In this case the sample, which was loaded with IL and subsequently activated at $60^{\circ}$ in an argon flow, was exposed to pure $\mathrm{EtOH}$ for $10 \mathrm{~min}$ and then again activated in an argon flow. In this case, the significant decrease in mass caused by the purging with $\mathrm{EtOH}$ indicates that, during this $\mathrm{EtOH}$ rinsing step, the IL leaves the SURMOF pores and is substituted by $\mathrm{EtOH}$. By activation, the $\mathrm{EtOH}$-filled pores are emptied.

We conclude, based on these QCM experiments, that the SURMOF can be loaded with the IL and hence that this is a suitable solvent/supporting electrolyte system.

HKUST-1 SURMOFs produced on both CMMT and TPMTA SAMs modified gold substrate were prepared by means of the spray method ${ }^{[23]}$ and characterized by IRRAS and XRD (Figures S4 and S5). Thereafter, the SURMOF HKUST-1 films were characterised by cyclic voltammetry employing ferrocene (Fc, see Figure 1c), an electroactive molecule widely used as standard in electrochemical investigations. ${ }^{[24]}$ Using a $5 \mathrm{mM}$ solution in IL/EtOH $(96 / 4, \mathrm{v} / \mathrm{v})$ the CVs of SURMOF HKUST-1 on either SAM1 (Figure 3a, in black) or SAM2 (Figure 3b, in black) were recorded in the range between $-0.35 \mathrm{~V}$ and $+0.55 \mathrm{~V}(v s \mathrm{Pt})$. Interestingly, the CVs are rather flat and, in particular, lack any features characteristic for the ferrocene/ferrocenium redox couple. This observation reveals that under the conditions and time frame of this experiment the redox active Fc species present in the solution do not reach the metal substrate to a significant extent. Combining the results from cyclic voltammetry with the ones from QCM, we conclude that the ionic liquid diffuses into the pores of the SURMOFs and, in combination with the confined space of the framework pores, essentially prevents the Fc molecules from moving across the HKUST-1 layer. We would like to note at this point that the CVs do not exhibit any features characteristic of the presence of defects acting as micro/nanoelectrodes ${ }^{[25]}$ which indicates that the SURMOF layer is homogeneous and free of substantial defects such as pinholes. How the presence of defects alters the CV is demonstrated by a sample which has been intentionally damaged by simply scratching across the sample (see Figure S6). The pronounced change in the diffusion properties is reflected by the transition from the flat $\mathrm{CV}$ of the pristine SURMOF layer towards the sigmoidal shape of a microelectrode, ${ }^{[25]}$ even though its shape is distorted as the scratch produces an ill-defined break-up of the SURMOF layer.

A completely different scenario occurs if the HKUST-1 SURMOF is loaded with ferrocene from the vapor phase ${ }^{[8 a, 26]}$ after activation of the MOF by soft annealing. The successful loading was proved by XRD and IRRAS. Showing the vibrational fingerprint of ferrocene the latter testifies the loading of the MOF with ferrocene (Figure S4). In the XRD $2 \theta$ scan (Figure S5), the decrease in the ratio of the (222) and (333) reflections is caused by the iron of the ferrocene, that is a quite strong $\mathrm{X}$-ray scatterer. This also indicates the presence of the molecule in the framework pores. However, even more important is that the crystallinity is not adversely affected by the ferrocene uptake, which is crucial for the interpretation of the electrochemical data.
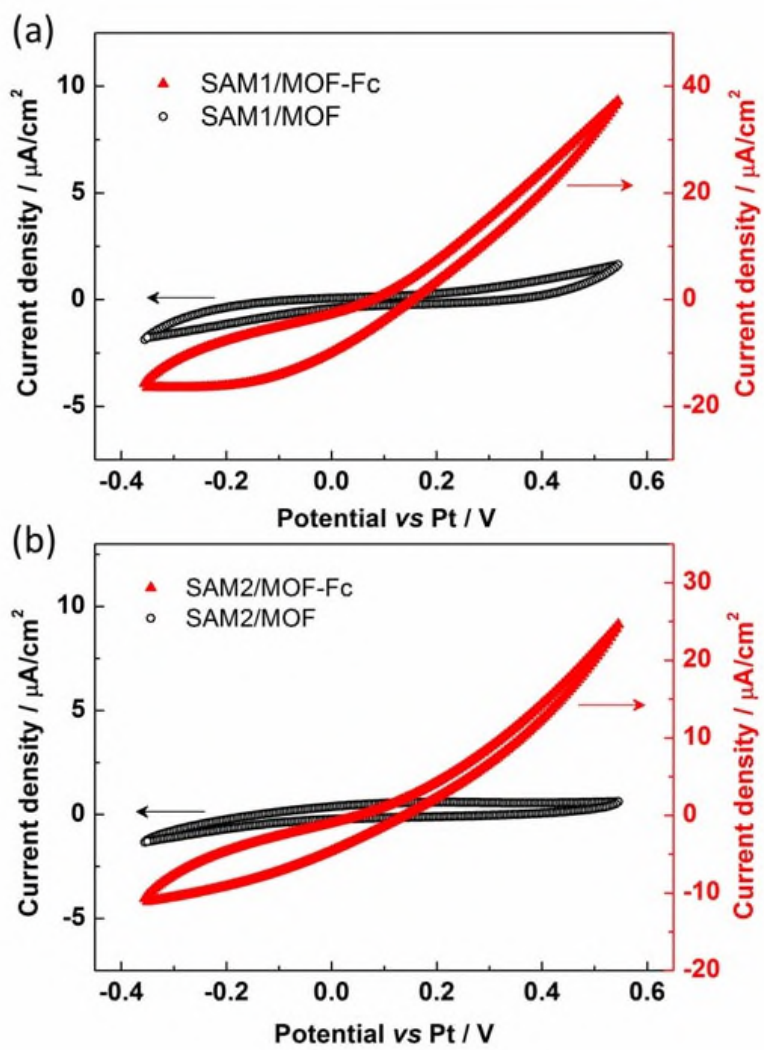

Figure 3. (a) $\mathrm{CV}$ of SAM1/MOF (black) and SAM1/MOF-Fc (red) recorded at a scan rate of $20 \mathrm{mV} / \mathrm{s}$. (b) CV of SAM2/MOF (black) and SAM2/MOF-Fc (red) recorded at a scan rate of $20 \mathrm{mV} / \mathrm{s}$.

The cyclic voltammograms (red curves in Figure 3) are pronouncedly different from the unmodified MOF as they now exhibit a quasi-linear increase in current density in the measured voltage range up to a value of $40 \mu \mathrm{A} / \mathrm{cm}^{2}$ at $+0.55 \mathrm{~V}$ at a scan rate of $20 \mathrm{mV} / \mathrm{s}$ for SAM1/MOF-Fc and analogously for SAM2/MOF. In principle, the difference in the CVs of the Fc loaded and unloaded SURMOF layers can be explained by two mechanisms. In one case the $\mathrm{Fc} / \mathrm{Fc}^{+}$redox reaction takes place at the MOF-metal interface and the current is mass transport limited due to a strongly hindered diffusion of $F c$ in the MOF compared to the bulk ionic liquid which would give rise to a grossly distorted CV. The other interpretation is a chargehopping mechanism where the Fc molecules remain localised in the pores of the MOF and mediate the electron transport across the layer. In this case the $\mathrm{Fc} / \mathrm{Fc}^{+}$redox reaction takes place at the MOF-IL interface. Given that the IL loaded MOF is impermeable for $\mathrm{Fc}$ as demonstrated above and the crystallinity 
of the Fc loaded MOF is not differing from the unloaded one, the first mechanism, which has been observed for post-synthetically modified surface anchored gels, ${ }^{[27]}$ is excluded. Furthermore, the essentially identical current density vs voltage dependency at different scan rates (Figure S7) contradicts a diffusion process. Since an analogous charge-hopping mechanism was also reported for HKUST-1 grown along the [001] direction on mercaptohexadecanoic acid (MHDA) SAM, ${ }^{[8 \mathrm{a}]}$ the present finding demonstrates that this is a general property of this MOF structure as it is not dependent on the SURMOF crystallographic orientation.

Looking at the CVs of the Fc loaded MOFs (Figures 3, S7) and the mechanically damaged one (Figure S6) there is a significant cathodic current in the reverse scan direction which indicates that diffusion of $\mathrm{Fc}^{+}$away from the MOF surface is incomplete. While the reasons for this are not established yet we tentatively explain this fact with the topography of the outer surface of the MOF which, as revealed by AFM (Figures S9/S10), exhibits a significant roughness and graininess. Nanoscopic kinks, pockets and grooves in the near surface region would, therefore, cause deviations from a free diffusion. Differences in the surface morphology could also account for small differences between the CVs.

A closer look at the quasi-linear plot of the current density $v s$ voltage shows two regimes differing in slope: In the anodic range (from $+0.14 \mathrm{~V}$ to $+0.55 \mathrm{~V}$ ) a resistance of $45.5 \mathrm{k} \Omega$ can be calculated for SAM1/MOF-Fc, and of $63 \mathrm{k} \Omega$ for SAM2/MOF-Fc (from the current density vs voltage dependence at a scan rate of $20 \mathrm{mV} / \mathrm{s}$, Table 1). In the cathodic range (from $-0.35 \mathrm{~V}$ to $+0.14 \mathrm{~V})$, where the linearity of the current density $v s$ voltage is less pronounced, a resistance of $236 \mathrm{k} \Omega$ for SAM1/MOF-Fc, and $224 \mathrm{k} \Omega$ for SAM2/MOF-Fc can be calculated (Table 1). This means that ferrocenium cations (formed in the anodic region and known to have a larger conductivity than neutral ferrocene $)^{[24 b]}$ are indeed generated inside the pores as result of the charge hopping taking place inside the framework. ${ }^{[28]}$

Table 1. Anodic and cathodic resistance and conductivity for SAM1/MOF-FC and SAM2/MOF-Fc. As thickness, values of $70 \pm 5 \mathrm{~nm}$ for SAM1/MOF-Fc and

\begin{tabular}{|l|c|c|c|c|}
\cline { 2 - 5 } \multicolumn{1}{c|}{} & \multicolumn{2}{c|}{$\begin{array}{c}\text { Anodic Range: } \\
\text { from 0.14V to } \mathbf{0 . 5 5 V}\end{array}$} & \multicolumn{2}{c|}{$\begin{array}{c}\text { Cathodic Range: } \\
\text { from }-\mathbf{0 . 3 5 V} \text { to }-\mathbf{0 . 1 4 V}\end{array}$} \\
\cline { 2 - 5 } \multicolumn{1}{c|}{} & $\mathbf{R} / \mathbf{~} \boldsymbol{\Omega}$ & $\boldsymbol{\sigma}_{\mathrm{a}} / \mathbf{S} \cdot \mathbf{c m}^{-1}$ & $\mathbf{R} / \mathbf{~} \boldsymbol{\Omega}$ & $\boldsymbol{\sigma}_{\mathrm{c}} / \mathbf{S} \cdot \mathbf{c m}^{-1}$ \\
\hline SAM1/MOF-Fc & 45.5 & $5.1 \pm 0.3 \times 10^{-10}$ & 236 & $9.9 \pm 0.15 \times 10^{-11}$ \\
\hline SAM2/MOF-Fc & 63 & $4.0 \pm 0.3 \times 10^{-10}$ & 224 & $1.1 \pm 0.05 \times 10^{-10}$ \\
\hline
\end{tabular}

$75 \pm 5 \mathrm{~nm}$ for SAM2/MOF-Fc have been used.

The conductivity of SURMOFs HKUST-1 was calculated from the resistance values after measuring the sample thickness by atomic force microscopy, as presented in Figure 4 . The topographic images show orientated crystallites of HKUST-1, likely as result of the LPE method chosen for the sample preparation. Nevertheless, these crystallites are sitting on top of a continuous and rather homogeneous MOF layer (see bottom right regions in Fig $4 \mathrm{c}$ and $4 \mathrm{~d}$ and explanation of film scratching in the experimental section), confirming the presence of a defect free film as inferred from cyclic voltammetry experiments.

The average vertical dimension of the crystallites is $\sim 20$ $\mathrm{nm}$ and $\sim 35 \mathrm{~nm}$ for SAM1/MOF-Fc and SAM2/MOF-Fc, respectively, whereas the underlying HKUST-1-Fc films exhibits a thickness of $\sim 70 \pm 5 \mathrm{~nm}$ for SAM1/MOF-Fc (see profile in Figure $4 \mathrm{e}$ ) and $\sim 75 \pm 5 \mathrm{~nm}$ for SAM2/MOF-Fc (Figure S11). Using the thickness of the homogeneous SURMOF layer as determined by AFM and the electrochemical cell area, the calculated conductivity values $(\sigma)$ at room temperature for each range result in $\sigma_{\mathrm{a}}=5.1 \pm 0.3 \times 10^{-10} \mathrm{~S} \cdot \mathrm{cm}^{-1}$ and in $\sigma_{\mathrm{c}}=9.9 \pm$ $0.15 \times 10^{-11} \mathrm{~S} \cdot \mathrm{cm}^{-1}$ for the anodic and cathodic ranges for SAM1/MOF-Fc, respectively (Table 1 ). The corresponding values for SAM2/MOF-FC are $\sigma_{\mathrm{a}}=4.0 \pm 0.3 \times 10^{-10} \mathrm{~S} \cdot \mathrm{cm}^{-1}$ and $\sigma_{\mathrm{c}}=1.1 \pm 0.05 \times 10^{-10} \mathrm{~S} \cdot \mathrm{cm}^{-1}$ (Table 1). These conductivities are somewhat lower than the ones provided in our previous report. ${ }^{[8 a]}$ These differences are attributed to variations in sample quality. Note that these conductivity values are a lower limit as only the homogenous and continuous HKUST-1 layers are considered. Indeed, contributions from the on top grown crystallites can also be considered. For the sake of simplicity, we take into account only the mean vertical size of the crystallites ( 20 nm and $\sim 35 \mathrm{~nm}$ for SAM1/MOF and SAM2/MOF, respectively) and not their contribution to the increase in surface area: by doing that an upper value of conductivity $\sigma_{a}=6.6 \times 10^{-}$ ${ }^{10} \mathrm{~S} \cdot \mathrm{cm}^{-1}$ and $\sigma_{a}=5.8 \times 10^{-10} \mathrm{~S} \cdot \mathrm{cm}^{-1}$, respectively, is obtained. These values are in good agreement with electric conductivity values measured for the SAM1/MOF-Fc films upon their integration in a $\mathrm{Hg}$-based tunneling junction. ${ }^{[29]}$
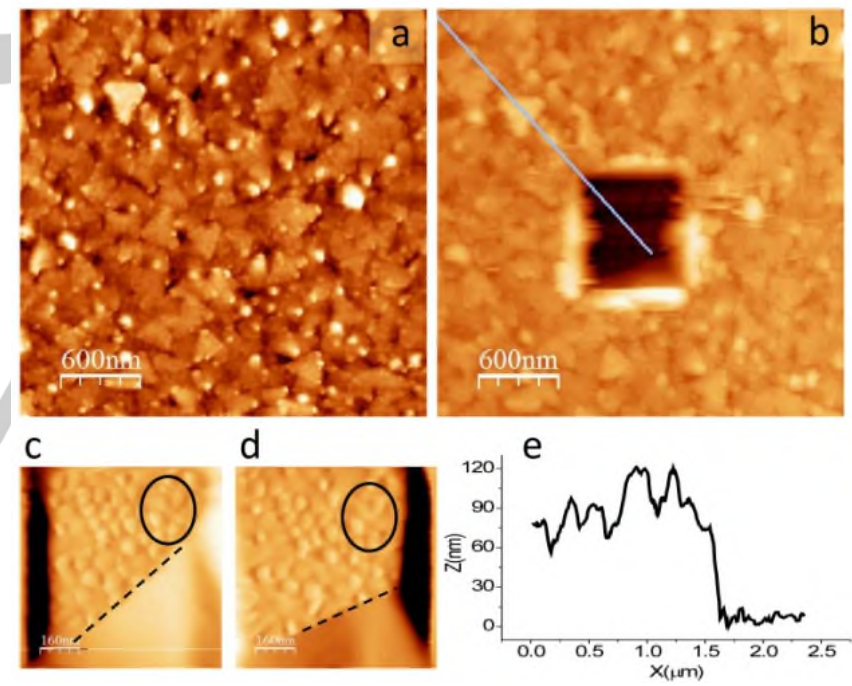

Figure 4. Topographic AFM images before (a) and after (b) local removal of the SURMOF on sample SAM1/MOF (imaging force $\sim 10 \mathrm{nN}$ ). Selected images (c and d) taken during the scratching ( $\sim 500 \mathrm{nN}$ load). Ellipses in (c) and (d) indicate the same areas of cleared substrate gold grains $(800 \mathrm{~nm} \times 800 \mathrm{~nm})$. Dashed lines indicate removal front (for details see text and $\mathrm{SI}$ ). The relief profile in (e) is along the line in (b) and illustrates the surface roughness and thickness of the SURMOF.

In conclusion, we have demonstrated that the spray based LPE method is suitable to produce crystalline and defect-free films of HKUST-1 on different SAMs and that the film quality is independent on crystallographic orientation of the film. In combination with other characterisation techniques, it is shown that by means of cyclic voltammetry the presence of defects or pinholes can be determined for SURMOFs covering 
macroscopic areas in a fast and straightforward fashion. Additionally, by means of cyclic voltammetry, we have also been able to gain further insight into the electronic properties of SURMOF HKUST-1 loaded with ferrocene. When ferrocene molecules are embedded inside the HKUST-1 pores, they are involved in a charge hopping mechanism that leads to a reversible ferrocene-ferrocenium redox process that takes place along the pores-immobilized guests and eventually triggers the oxidation of ferrocene electrolyte at the IL/MOF interface.

Following the presented strategy, electrochemical characterization of other SURMOFs with ferrocene and other electroactive molecules is in progress and will help us to shed light on the relation between SURMOF crystalline orientation and pore size, morphology and lateral homogeneity, and their electrochemical behaviour. In addition, we plan to carry out further experiments to elucidate the role of the used supporting electrolyte as well as of the electroactive guests immobilised in the SURMOF.

\section{Experimental Section}

\section{SAM Preparation}

SAMs were formed on gold substrates $(100 \mathrm{~nm}$ of $\mathrm{Au}$ on Si substrate, with a $5 \mathrm{~nm}$ Ti adhesion layer; purchased from Georg Albert PVD) or on QCM sensors (Au coated, purchased from LOT-ORIEL) by immersion into solutions of CMMT (9-carboxy-10-(mercaptomethyl) triptycene) and TPMTA (4'-carboxyterphenyl-4-methanethiol), as described in the literature. ${ }^{[14]}$

\section{Preparation of SURMOF HKUST-1 and characterization by means of} out-of-plane XRD and IRRAS

The HKUST-1 SURMOFs on CMMT and TPMTA, SAM1/MOF and SAM2/MOF, respectively, were prepared in a stepwise fashion using the spray method (copper acetate mono-hydrate, $1 \mathrm{mM}$ in ethanol; $1,3,5$ benzenetricarboxylic acid, $0.1 \mathrm{mM}$ in ethanol; both purchased from Sigma Aldrich and used without further purification). This method ${ }^{[23]}$ is a modification of the well described LPE ${ }^{[139]}$ method that drastically reduces the time needed for sample preparation, without affecting neither the crystallinity nor the definition of the crystallographic orientation. Indeed, out-of-plane XRD (Bruker D8-Advance diffractometer) patterns show a good crystallographic orientation for SAM1/MOF (Figure S5a) and a less pronounced orientation for SAM2/MOF (Figure S5b). The loading with ferrocene (Fc, from Sigma Aldrich and used without further purification) was done by exposure of SAM1/MOF and SAM2/MOF to Fc vapours in a sealed vial ${ }^{[8 a]}$ for a time of typically $64 \mathrm{~h}$ after activation of the samples (20 minutes at $60^{\circ} \mathrm{C}$ ) in a vacuum oven which removes residual solvent molecules trapped in the pores. The resulting Fc loaded samples, named herein SAM1/MOF-FC and SAM2/MOF-Fc respectively, were characterized by IRRAS and out-of-plane XRD (Figures S4 and S5, in red).

\section{Quartz Crystal Microbalance}

A quartz crystal microbalance (QCM, of type E4 from Q-Sense) was used for quantifying the IL uptake by the SURMOF. The solutions were pumped at a flow rate of $0.5 \mathrm{ml} / \mathrm{min}$ through the QCM cell; the argon flow rate was $100 \mathrm{ml} / \mathrm{min}$. The loading experiments as well as the activation in argon were performed at a temperature of $60^{\circ} \mathrm{C}$.

\section{Electrochemical measurements}

Cyclic voltammetry (CV) was carried out in a home-built electrochemical cell using a standard three electrode set-up, with reference (placed in a
Luggin capillary) and counter electrodes made from platinum wires (diameter $0.25 \mathrm{~mm}$, Advent Research Materials Ltd). The area of the working electrode was $0.3 \mathrm{~cm}^{2}$. Measurements of SAM1, SAM1/MOF, SAM1/MOF-Fc, SAM2, SAM2/MOF, SAM2/MOF-Fc were done under continuous argon flow. A PalmSens potentiostat was used. As supporting electrolyte, the aprotic ionic liquid (IL) 1-butyl-3-methylimidazolinium bis(trifluoronethylsulfonyl)-imide ([BMIM] $\left[\mathrm{NTf}_{2}\right]$ ) from io-li-tec, (structure presented in Figure 1(c)), was chosen and ferrocene (5 mM) was dissolved by adding a small amount of ethanol (4/96 EtOH/[BMIM][NTf 2$])$. CVs were acquired by exposing the sample to the solution of ferrocene (Figure 1c) in [BMIM][NTf $f_{2}$. The potential window was kept constant for all the experiments (from -0.35 to $+0.55 \mathrm{~V}$ vs Pt) while the scan rate was varied $(20 \mathrm{mV} / \mathrm{s}, 50 \mathrm{mV} / \mathrm{s}, 100 \mathrm{mV} / \mathrm{s})$. After the electrochemical experiments the samples were routinely checked by XRD, and no loss in in the crystalline structure of the SURMOF could be observed (see Figure S2).

\section{Atomic force microscopy measurements}

As done previously for similar systems, the thickness of the samples after ferrocene loading was accurately determined by atomic force microscopy (AFM) employing the scratching method. ${ }^{[16 \mathrm{~b}]}$ The AFM images (Figure 4 and Figure S10) clearly show on both template surfaces SAM1 and SAM2 the presence of oriented crystallites of HKUST-1. As seen in Figure 4a for SAM1/MOF-Fc, though randomly distributed in azimuth, the crystallites exhibit a flat top surface with a clear triangular shape indicative of a (111) orientation, in agreement with the XRD data shown in Figure S5. Details of the morphology and orientation of the crystallites for SAM2/MOF are shown in Figure S10. Scratching experiments (details in SI) were performed on the very same surface region (see Figure 4b) to remove the grown SURMOF until the underlying gold substrate is completely uncovered (Figure 4, and Figures S9 and S11). Figure 4c and $4 \mathrm{~d}$ correspond to images taken at the central part of the region in $4 \mathrm{~b}$ during the scratching and serve to illustrate the process. The small grains of the underlying gold substrate are clearly recognized in the cleared areas. To be used as a reference, the same gold grains have been circled in both images and a dashed line has been drawn in each image to mark the material removal front. Note the movement of the front between $4 \mathrm{c}$ and $4 \mathrm{~d}$. The AFM measurements were performed under low humidity conditions ( $<5 \% \mathrm{RH}$, obtained by a continuous $\mathrm{N}_{2}$ flux) using a commercial head and electronics from Nanotec. ${ }^{[30]} \mathrm{Si}$ probes from Nanosensors with an intermediate spring constant $(k=2.8 \mathrm{~N} / \mathrm{m})$ were used. The imaging force was set to below $10 \mathrm{nN}$ while a force in the range of $400-700 \mathrm{nN}$ was applied to remove the MOF (see SI for more details).

\section{Acknowledgements}

VM acknowledges the EU for the funding through the granting of a Marie Curie Intra-European Fellowship (FP7-PEOPLE-2011; MOLSURMOF301110). JL acknowledges the program of China Scholarship Council (CSC) for financial support. VM, JL and CW acknowledge A. Terfort for supplying the thiol derivatives. MP and $\mathrm{CO}$ acknowledge financial support from the Spanish Government through grants projects MAT201347869-C4-1-P and SEV-2015-0496 and Generalitat de Catalunya through grant project 2014 SGR 501. VM and JL thank P.G. Weidler for the useful help in the analysis of XRD data.

Keywords: Surface anchored metal-organic frameworks • HKUST-1 • ferrocene $\cdot$ cyclic voltammetry $\bullet$ ionic liquid $\cdot$ defectfree film 
[1] a) S. I. Noro, S. Kitagawa, in The Supramolecular Chemistry of Organic-Inorganic Hybrid Materials, ed. K. Rurack and R. MartínezMáñez, John Wiley \& Sons, Inc. 2010, pp. 235-269; b) S. L. James, Chem. Soc. Rev. 2003, 32, 276-288.

[2] a) H. Furukawa, N. Ko, Y. B. Go, N. Aratani, S. B. Choi, E. Choi, A. O. Yazaydin, R. Q. Snurr, M. O'Keeffe, J. Kim, O. M. Yaghi, Science 2010, 329, 424-428; b) D. Zhao, D. J. Timmons, D. Q. Yuan, H. C. Zhou, Acc. Chem. Res. 2011, 44, 123-133; c) F. A. A. Paz, J. Klinowski, S. M. F Vilela, J. P. C. Tome, J. A. S. Cavaleiro, J. Rocha, Chem. Soc. Rev. 2012, 41, 1088-1110.

[3] a) P. R. McGonigal, P. Deria, I. Hod, P. Z. Moghadam, A-J Avestro, N. E. Horwitz, I. C. Gibbs-Hall, A. K. Blackburn, D. Chen, Y. Y. Botros, M. R. Wasielewski, R. Q. Snurr, J. T. Hupp, O. K. Farha, J. F. Stoddart, PNAS. 2015, 112, 11161-11168; b) B. Liu, H. Shioyama, T. Akita, Q. Xu J. Am. Chem. Soc. 2008, 130, 5390-5391; c) M. Meilikhov, K. Yusenko, A. Torrisi, B. Jee, C. Mellot-Draznieks, A. Poppl, R. A. Fischer, Angew. Chem., Int. Ed. 2010, 49, 6212-6215.

[4] P. Falcaro, R. Ricco, C. M. Doherty, K. Liang, A. J. Hill, M. J. Styles, Chem. Soc. Rev. 2014, 43, 5513-5560.

[5] a) M. Usman, S. Mendiratta, K-L. Lu, ChemElectroChem. 2015, 2, 786788; b) A. Morozan, F. Jaouen, Energy Environ. Sci. 2012, 5, 9269 9290; c) M. D. Allendorf, A. Schwartzberg, V. Stavila, A. A. Talin, Chem. Eur. J. 2011, 17, 11372-11388.

[6] a) Z. Y. Zhang, H. Yoshikawa, K. Awaga, J. Am. Chem. Soc. 2014, 136, 16112-16115; b) D. F. Wu, Z. Y. Guo, X. B. Yin, Q. Q. Pang, B. B. Tu, L. J. Zhang, Y. G. Wang, Q. W. Li, Adv. Mater. 2014, 26, 3258-3262.

[7] a) S. Takaishi, M. Hosoda, T. Kajiwara, H. Miyasaka, M. Yamashita, Y. Nakanishi, Y. Kitagawa, K. Yamaguchi, A. Kobayashi, H. Kitagawa, Inorg. Chem. 2009, 48, 9048-9050; b) Y. Kobayashi, B. Jacobs, M. D. Allendorf, J. R. Long, Chem. Mater. 2010, 22, 4120-4122; c) K. Hirai, H. Uehara, S. Kitagawa, S. Furukawa, Dalton Trans. 2012, 41, 3924-3927; d) T. C. Narayan, T. Miyakai, S. Seki, M. Dinca, J. Am. Chem. Soc. 2012, 134, 12932-12935; e) D. Umeyama, S. Horike, M. Inukai, T. Itakura, S. Kitagawa, J. Am. Chem. Soc. 2012, 134, 12780-12785.

[8] a) A. Dragasser, O. Shekhah, O. Zybaylo, C. Shen, M. Buck, C. Wöll, D. Schlettwein, Chem. Commun. 2012, 48, 663-665; b) A. A. Talin, A. Centrone, A. C. Ford, M. E. Foster, V. Stavila, P. Haney, R. A. Kinney, V. Szalai, F. El Gabaly, H. P. Yoon, F. Leonard, M. D. Allendorf, Science 2014, 343, 66-69.

[9] V. Stavila, A. A. Talin, M. D. Allendorf, Chem. Soc. Rev. 2014, 43, 5994-6010.

[10] J. Liu, W. Zhou, J. Liu, I. Howard, G. Kilibarda, S. Schlabach, D. Coupry, M. Addicoat, S. Yoneda, Y. Tsutsui, T. Sakurai, S. Seki, Z. Wang, P. Lindemann, E. Redel, T. Heine, C. Wöll, Angew. Chem., Int. Ed. 2015, $54,7441-7445$.

[11] a) C. H. Hendon, D. Tiana, A. Walsh, Phys. Chem. Chem. Phys. 2012, 14, 13120-13132; b) K. T. Butler, C. H. Hendon, A. Walsh, J. Am. Chem. Soc. 2014, 136, 2703-2706.

[12] a) W. Zhou, C. Wöll, L. Heinke, Materials, 2015, 8, 3767-3775; b) L. Heinke, Z. G. Gu, C. Wöll, Nat. Commun. 2014, 5, 4562.

[13] a) J-L. Zhuang, A. Terfort, C. Wöll, Coord. Chem. Rev. 2016, 307, 391424; b) L. Heinke, M. Tu, S. Wannapaiboon, R. A. Fischer, C. Wöll,
Microporous Mesoporous Mater. 2015, 216, 200-215; c) H. Al-Kutubi, J. Gascon, E.J.R. Sudhölter, L. Rassaei, ChemElectroChem. 2015, 2, 462-474; d) C. R. Wade, M. Y. Li, M. Dinca, Angew. Chem., Int. Ed. 2013, 52, 13377-13381; e) N. Campagnol, T. Van Assche, T. Boudewijns, J. Denayer, K. Binnemans, D. De Vos, J. Fransaer, J. Mater. Chem. A 2013, 1, 5827-5830; f) T. R. C. Van Assche, G. Desmet, R. Ameloot, D. E. De Vos, H. Terryn, J. F. M. Denayer, Microporous Mesoporous Mater. 2012, 158, 209-213; g) O. Shekhah, J. Liu, R. A. Fischer, C. Wöll, Chem. Soc. Rev. 2011, 40, 1081-1106; h) A. Schoedel, C. Scherb, T. Bein, Angew. Chem., Int. Ed. 2010, 49, 7225-7228; i) O. Shekhah, H. Wang, D. Zacher, R. A. Fischer, C. Wöll, Angew. Chem., Int. Ed. 2009, 48, 5038-5041; i) C. Scherb, A. Schodel, T. Bein, Angew. Chem., Int. Ed. 2008, 47, 5777-5779.

[14] J. X. Liu, O. Shekhah, X. Stammer, H. K. Arslan, B. Liu, B. Schupbach, A. Terfort, C. Wöll, Materials 2012, 5, 1581-1592.

[15] Z. Wang, J. Liu, B. Lukose, Z. Gu, P. G. Weidler, H. Gliemann, T. Heine, C. Wöll, Nano Lett. 2014, 14, 1526-1529.

[16] a) C. Munuera, O. Shekhah, H. Wang, C. Wöll, C. Ocal, Phys. Chem. Chem. Phys. 2008, 10, 7257-7261; b) O. Shekhah, H. Wang, M. Paradinas, C. Ocal, B. Schupbach, A. Terfort, D. Zacher, R. A. Fischer, C. Wöll, Nat. Mater. 2009, 8, 481-484.

[17] S. S. Y. Chui, S. M. F. Lo, J. P. H. Charmant, A. G. Orpen, I. D. Williams, Science 1999, 283, 1148-1150.

[18] R. D. Rogers, K. R. Seddon, Science 2003, 302, 792-793.

[19] L. Heinke, C. Wöll, Phys. Chem. Chem. Phys. 2013, 15, 9295-9299.

[20] I. Goubaidoulline, G. Vidrich, D. Johannsmann, Anal. Chem. 2005, 77, 615-619.

[21] a) S. J. Zhang, N. Sun, X. Z. He, X. M. Lu, X. P. Zhang, J. Phys. Chem. Ref. Data 2006, 35, 1475-1517; b) J. Jacquemin, P. Husson, A. A. H. Padua, V. Majer, Green Chem. 2006, 8, 172-180.

[22] G. Sauerbrey, Zeitschrift Fur Physik 1959, 155, 206-222.

[23] H. K. Arslan, O. Shekhah, J. Wohlgemuth, M. Franzreb, R. A. Fischer, C. Wöll, Adv. Funct. Mater. 2011, 21, 4228-4231.

[24] a) A. D. Le, L. Yu, J. Electrochem. Soc. 2011, 158, F10-F14; b) D. O. Cowan, Y. Sasaki, J. Park, C. U. Pittman, Mukherje.Tk, N. A. Diamond, J. Am. Chem. Soc. 1972, 94, 5110-5112.

[25] J. Heinze, Angew. Chemie Int. Ed. 1993, 32, 1268-1288.

[26] M. J. S. Monte, L. M. N. B. F. Santos, M. Fulem, J. M. S. Fonseca, C. A. D. Sousa, J. Chem. Eng. Data 2006, 51, 757-766.

[27] V. Mugnaini, M. Tsotsalas, F. Bebensee, S. Grosjean, A. Shahnas, S. Brase, J. Lahann, M. Buck, C. Wöll, Chem. Commun. 2014, 50, 1112911131.

[28] Note: There is a typographical error in the unit of the resistance provided for the HKUST-1 SURMOF in ref. 8a. Instead of $31 \mathrm{M} \Omega$ it should read $31 \mathrm{k} \Omega$. The conductivity values provided in this ref. are correct.

[29] J. Liu, T. Wachter, A. Irmler, P. G. Weidler, H. Gliemann, F. Pauly, V. Mugnaini, M. Zharnikov, C. Wöll, ACS Appl. Mater. Interfaces 2015, 7, 9824-9830.

[30] I. Horcas, R. Fernandez, J. M. Gomez-Rodriguez, J. Colchero, J. Gomez-Herrero, A. M. Baro, Rev. Sci. Instrum. 2007, 78, 013705. 
Jianxi Liu, ${ }^{[a]}$ Markos

Paradinas, ${ }^{[b],[c]}$ Lars Heinke, ${ }^{[a]}$

Manfred Buck, ${ }^{[d]}$ Carmen

Ocal, ${ }^{[b]}$ Veronica Mugnaini, ${ }^{*}[a],[e]$

Christof Wöl/[ ${ }^{* a]}$

Page No. - Page No.

Film quality and electronic properties of a surfaceanchored metal-organic framework as revealed by a multi-technique approach

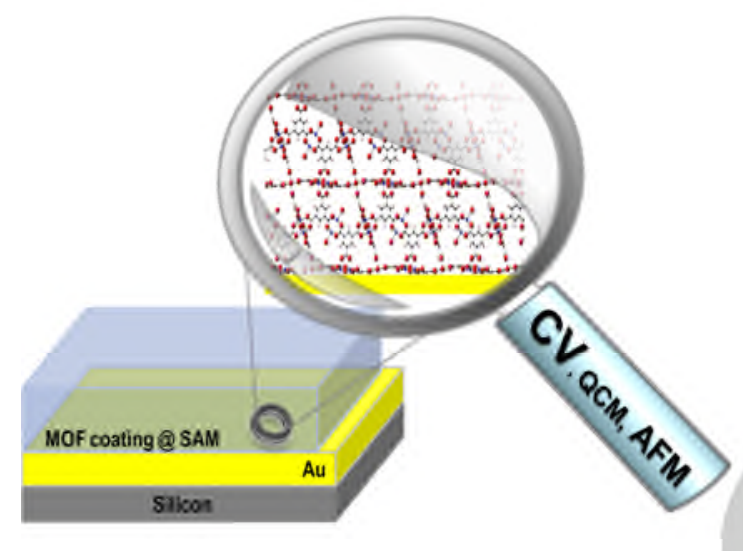

The film quality matters: Metal-organic frameworks have recently been listed as most promising candidates for electronic applications. For their implementation in working devices, film quality (i.e. absence of pinholes and defects) as well as electronic properties have to be investigated. We use a multi-technique approach based on cyclic voltammetry (CV) supported by quartz-crystal microbalance (QCM) and atomic force microscopy (AFM) to get valuable information on the film quality. Additionally, we have studied the changes in electric conductivity of the MOF films upon filling their pores with a small electroactive molecule, ferrocene. 